\title{
Acceptability of HIV self-testing to support pre-exposure prophylaxis among female sex workers in Uganda and Zambia: results from two randomized controlled trials
}

Katrina F. Ortblad' ${ }^{1}$, Michael M. Chanda², Daniel Kibuuka Musoke ${ }^{3}$, Thomson Ngabirano ${ }^{4}$, Magdalene Mwale ${ }^{2}$, Aidah Nakitende ${ }^{3}$, Steven Chongo ${ }^{2}$, Nyambe Kamungoma2 ${ }^{2}$, Catherine Kanchele², Till Bärnighausen ${ }^{5,6,7}$ and Catherine E. Oldenburg ${ }^{8,9,10^{*}}$ (i)

\begin{abstract}
Background: HIV pre-exposure prophylaxis (PrEP) is highly effective for prevention of HIV acquisition, but requires HIV testing at regular intervals. Female sex workers (FSWS) are a priority population for HIV prevention interventions in many settings, but face barriers to accessing healthcare. Here, we assessed the acceptability of HIV self-testing for regular HIV testing during PrEP implementation among FSWs participating in a randomized controlled trial of HIV self-testing delivery models.

Methods: We used data from two HIV self-testing randomized controlled trials with identical protocols in Zambia and in Uganda. From September-October 2016, participants were randomized in groups to: (1) direct delivery of an HIV self-test, (2) delivery of a coupon, exchangeable for an HIV self-test at nearby health clinics, or (3) standard HIV testing services. Participants completed assessments at baseline and 4 weeks. Participants reporting their last HIV test was negative were asked about their interest in various PrEP modalities and their HIV testing preferences. We used mixed effects logistic regression models to measure differences in outcomes across randomization arms at four weeks.

Results: At 4 weeks, 633 participants in Zambia and 749 participants in Uganda reported testing negative at their last HIV test. The majority of participants in both studies were "very interested" in daily oral PrEP (91\% Zambia; 66\% Uganda) and preferred HIV self-testing to standard testing services while on PrEP (87\% Zambia; 82\% Uganda). Participants in the HIV self-testing intervention arms more often reported preference for HIV self-testing compared to standard testing services to support PrEP in both Zambia $(P=0.002)$ and Uganda $(P<0.001)$.
\end{abstract}

Conclusion: PrEP implementation programs for FSW could consider inclusion of HIV self-testing to reduce the clinicbased HIV testing burden.

Trial registration: ClinicalTrials.gov NCT02827240 and NCT02846402.

Keywords: Pre-exposure prophylaxis, Female sex workers, HIV self-testing, Zambia, Uganda

\footnotetext{
* Correspondence: catherine.oldenburg@ucsf.edu

${ }^{8}$ Francis I. Proctor Foundation, University of California San Francisco, 513

Parnassus Ave, Room S334J, San Francisco, CA, USA

${ }^{9}$ Department of Ophthalmology, University of California, San Francisco, San

Francisco, USA

Full list of author information is available at the end of the article
}

(c) The Author(s). 2018 Open Access This article is distributed under the terms of the Creative Commons Attribution 4.0 International License (http://creativecommons.org/licenses/by/4.0/), which permits unrestricted use, distribution, and reproduction in any medium, provided you give appropriate credit to the original author(s) and the source, provide a link to the Creative Commons license, and indicate if changes were made. The Creative Commons Public Domain Dedication waiver (http://creativecommons.org/publicdomain/zero/1.0/) applies to the data made available in this article, unless otherwise stated. 


\section{Background}

HIV pre-exposure prophylaxis (PrEP) is highly efficacious for the prevention of HIV acquisition in men who have sex with men [1] and heterosexual serodiscordant couples $[2,3]$. Although few HIV seroconversions have been documented in the context of PrEP implementation programs in the United States [4-7], there is concern related to the development of resistance for individuals who acquire HIV while taking PrEP [7]. Routine HIV testing is therefore a core component of patient management in PrEP programs. The World Health Organization [8] and the Centers for Disease Control and Prevention [9] recommend quarterly HIV testing for all individuals taking PrEP. However, frequent HIV testing can be burdensome to some PrEP patients, particularly for those who face logistical barriers to accessing healthcare [10].

HIV self-testing allows individuals to test for HIV at the time and place of their choosing, and may reduce some barriers to accessing regular HIV testing. In the context of PrEP implementation programs, HIV self-testing might be a useful complement to clinic-based testing [11]. For example, if the sensitivity and specificity of HIV self-testing is adequate for people taking PrEP [12], HIV self-testing could be used to screen for HIV instead of clinic visits for some time points. HIV self-testing has generally been shown to be acceptable $[13,14]$ and has been implemented in diverse populations of users [15].

Female sex workers (FSW) in Sub-Saharan Africa are disproportionately affected by the HIV epidemic [16]. PrEP has the potential to substantially alter the course of the HIV epidemic among FSW, particularly as a user-controlled prevention intervention that does not require negotiation with a partner, such as male condom use [17]. FSW often receive a higher price for engaging in condomless sex, and thus may be economically disincentivized from using male condoms in particular [18]. PrEP may therefore be a beneficial tool for FSW in some contexts [19]. A demonstration project of PrEP integration into routine care for FSW in South Africa demonstrated good uptake but suboptimal retention in PrEP care [20]. Barriers to accessing routine HIV testing exist for many populations of FSW, including logistical barriers such as timing of clinic hours and interpersonal barriers such as anticipated stigma from healthcare workers [21-24], which may contribute to poor retention in PrEP care.

Here, we report PrEP acceptability among FSW participating in two trials of oral HIV self-testing delivery models in Zambia [25] and Uganda [26], and the acceptability of HIV self-testing in the context of PrEP use.

\section{Methods}

\section{Participants and procedures}

We conducted two separate three-arm cluster randomized trials of HIV self-testing delivery models for FSWs in three Zambian transit towns (ClinicalTrials.gov NCT02827240)
[25] and urban Uganda (ClinicalTrials.gov NCT02846402) [26]. The two trials followed identical protocols and measured identical outcomes at each time point [27]. The present analysis is a pre-specified secondary analysis from each parent trial.

Trained peer educators each recruited 6 participants in Zambia and 8 in Uganda. In Zambia, participants were recruited in Livingstone, Kapiri Mposhi, and Chirundu, all transit towns. In Uganda, participants were recruited in Kampala, the capital city. In each country, individuals were eligible for participation if they were at least 18 years of age, reported exchanging sex for money or goods at least once in the previous month, self-reported an HIV negative status and no recent $(<3$ months) HIV test or an unknown HIV serostatus, and were permanent residence in their town/city of recruitment.

All participants completed four peer educator visits at weeks $0,2,6$, and 10, and three study visits at baseline prior to randomization and 4 and 16 weeks after the first peer educator visit. We restricted our analysis to participants who self-reported that the results of their most recent HIV test were negative at the 4-week study visit. PrEP-related outcomes were only measured at the 4-week study visit, and thus no 16-week data were included in this analysis.

\section{Randomization}

Participants were randomized in groups of one peer educator and 6 participants in Zambia and 8 participants in Uganda. Groups were randomized in a 1:1:1 fashion to one of three randomization arms: 1) direct distribution of the HIV self-test kit from the peer educator to the participant (direct delivery), 2) distribution of a coupon from the peer educator to the participant that could be used to collect the HIV self-test from an existing health facility (coupon delivery), or 3) referral from the peer educator to existing and free of charge standard HIV testing facilities (standard of care). The randomization list was generated in $\mathrm{R}$ (Version 3.3.1, The R Foundation for Statistical Computing, Vienna, Austria) in random blocks of size 3, 6, and 9. A separate randomization list was generated for the Zambia and Uganda studies. In both countries, the randomized study assignments were placed in opaque envelopes that were opened by a peer educator and a study staff member once each peer educator group enrolled its target number of participants.

\section{Interventions}

All participants completed four peer educator visits, at weeks $0,2,6$, and 10 . At baseline, the peer educator visit was done in a group setting and consisted of HIV prevention counseling, distribution of male condoms, and discussion of where participants could go for HIV testing. In the HIV self-testing arms, participants were 
additionally given either an HIV self-test kit (direct delivery arm) or coupon that could be used for collection of an HIV self-test at a participating pharmacy or health clinic (coupon delivery). We used the OraQuick Rapid HIV-1/2 Antibody Test (OraSure Technologies, Bethlehem, PA), which has been shown to have a sensitivity of $98.7 \%$ and specificity of $99.8 \%$ in Zambia under field conditions [28]. Subsequent peer educator visits were one-on-one, and consisted of screening for adverse events, distribution of male condoms, and additional discussion related to HIV testing. Participants in the HIV self-testing arms were also able to ask their peer educator if they needed any help with HIV self-testing. At the fourth peer educator visit (week 10), participants in the HIV self-testing arms received a second HIV self-test or coupon for collection of an HIV self-test at a health clinic.

\section{Measures}

Participants completed three surveys using computer-assisted personal interview with a trained research assistant. Interviews occurred at baseline prior to randomization and weeks 4 and 16 following the first peer educator visit.

\section{Demographic characteristics}

At baseline, participants were asked their age, literacy (if they could read and write), if they owned a mobile phone, monthly income (Kwacha in Zambia or Ugandan Shillings in Uganda), and if they had a primary partner (e.g., husband or boyfriend who is not a client). Participants were also asked the age at which they began working in sex work. The number of years participants engaged in sex work was calculated as the difference in their current age and the age at which they reported starting working in sex work. Participants were additionally asked to report the number of clients they had on an average working night, and how many of these they used a condom (male or female) with on average. Participants were categorized as inconsistently using condoms (male or female) with clients if the number of clients with whom they used a condom (male or female) on an average night was less than their average number of clients per night. Finally, participants were asked to estimate how likely it was, on a 10-point ladder scale, that they would acquire HIV in the next year.

\section{PrEP acceptability}

At the 4-week visit, research assistants read a brief script to participants that introduced daily oral PrEP. The script described daily oral PrEP as a new method for preventing HIV for people who do not have HIV but are at risk of getting it. Participants were told by the research assistant that PrEP has been shown to reduce the risk of HIV infection when taken consistently. Participants were asked how interested they were in taking daily oral PrEP, and their responses were categorized either as "very interested" or less. Injectable PrEP, PrEP in the form of a vaginal microbicide, and a vaginal ring were then introduced to participants as forms of PrEP that were being studied. Injectable PrEP was described as a shot that is given every 3 months. Vaginal microbicides were described as gels or lubricants that were inserted into the participant's vagina. Vaginal rings were described as rings that are inserted into the vagina that lasts for one month. Participants were asked how interested they were in taking each PrEP modality, interest was again categorized as "very interested" or less.

\section{Acceptability of HIV self-testing during PrEP use}

Participants were told by the research assistant that individuals taking PrEP should be tested for HIV every 3 months. Participants were then asked if they would be willing to test for HIV every three months. This question did not specify which type of testing (e.g., self-test or standard facility-based testing). Finally, participants were asked if they would prefer standard HIV testing at a clinic or HIV self-testing for testing while taking PrEP.

\section{Statistical analysis}

Descriptive characteristics were calculated with proportions for categorical variables and medians and interquartile ranges (IQR) for continuous variables. We calculated the proportion of participants who responded that they would be "very willing" to use each PrEP modality across all study arms. To determine whether exposure to the HIV self-testing intervention led to increased willingness to test for HIV as part of a PrEP program or preference for HIV self-testing versus standard testing, we used mixed effects logistic regression model with a fixed effect for study arm and a random effect for peer educator group. All analyses were conducted separately for the Uganda and Zambia studies. All $P$-values were two-sided and analyses were run in Stata 14.1 (StataCorp, College Station, TX).

\section{Results}

From September-October of 2016, 965 participants enrolled in the Zambia trial and 960 participants enrolled in the Uganda trial (Fig. 1). At the four-week study visit, retention of study participants in the Zambia trial was $92 \%(886 / 965)$ and retention of study participants in the Uganda trial was $96 \%$ (926/960). Of these participants, $71 \%(633 / 886)$ in Zambia and $81 \%(749 / 926)$ in Uganda reported testing HIV-negative at their last HIV test and were included in our study population.

Table 1 shows the demographic characteristics of participants in our study populations at the baseline visit. Participants in Zambia were slightly younger than participants in Uganda. In Zambia, the mean age of study participants was 24 years (interquartile range [IQR] 21 to 29), while in 


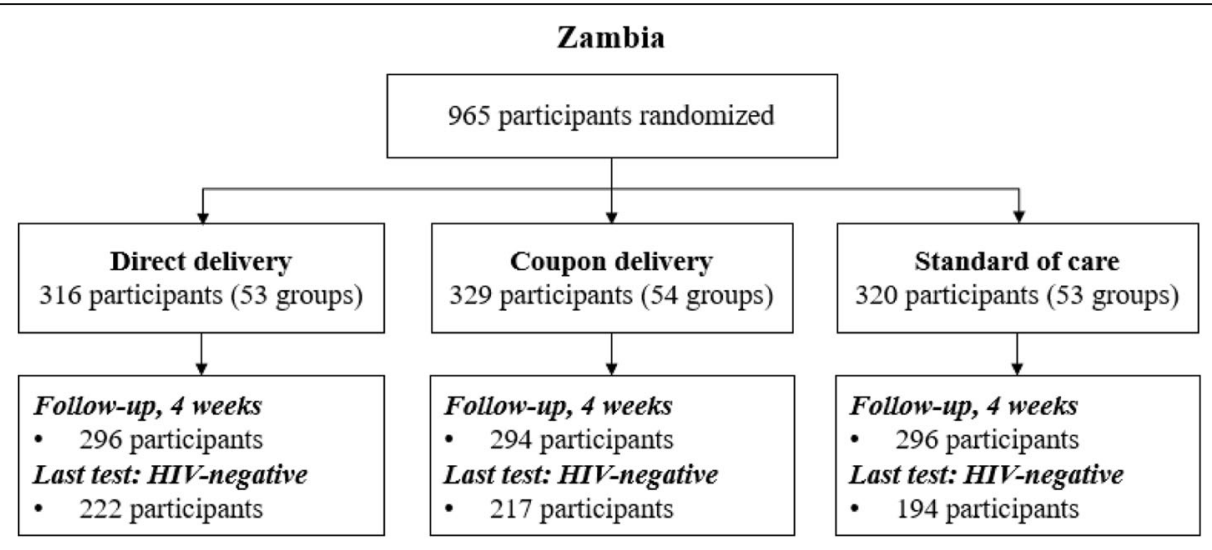

Uganda

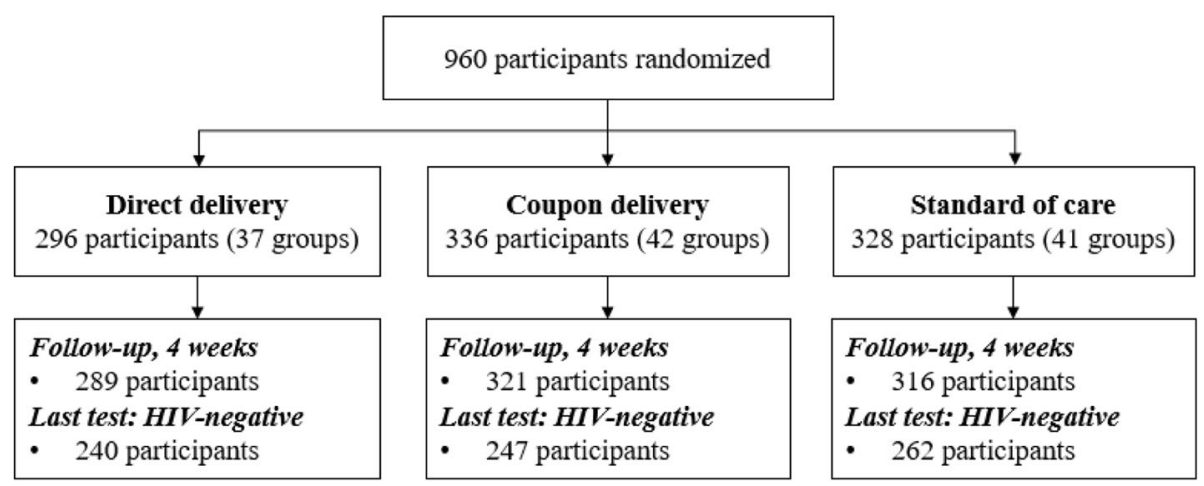

Fig. 1 Flow of participants included in the study in both Zambia and Uganda

Uganda the median age of study participants was 28 years (IQR 24 to 32). The majority of participants in both studies had a primary sexual partner, self-reported the ability to read and write and owned a mobile phone. Compared to participants in Uganda, those in Zambia tended to be newer to sex work, had a higher prevalence of inconsistent condom use with clients, and perceived themselves at greater risk of acquiring HIV in the next year. There were no statistically significant differences in demographic characteristics at baseline across the three randomization arms in both Zambia and Uganda.

PrEP acceptability was high among participants in both studies. Figure 2 shows the percentage of participants that reported being "very interested" in the different PrEP modalities. Almost all participants in Zambia and the majority of participants in Uganda reported being "very interested" in daily oral PrEP. The vast majority of participants in both settings also reported being "very interested" in quarterly injectable PrEP. Interest in vaginally applied PrEP was less common. Roughly half of participants in both settings said they would be "very interested" in PrEP as a vaginal gel, and only $40 \%$ of participants in Zambia and $21 \%$ of participants in Uganda said they would be "very interested" in PrEP as a vaginal ring.
Almost all participants in both study sites (99\% Zambia; 97\% Uganda) reported that they would be willing to test for HIV every three months while taking PrEP. The percentage of participants that reported willingness to take PrEP by randomization arm and study site is shown in Table 2. The different HIV self-testing delivery models did not significantly affect participants' willingness to test for HIV every three months while on PrEP in either Zambia $(P=0.64)$ or Uganda $(P=0.19)$.

The different HIV self-testing delivery models did, however, significantly affect participants' preference for HIV self-testing over standard HIV testing services in both Zambia and Uganda. Figure 3 shows the percentage of participants by randomization arm and study site that reported a preference for HIV self-testing or standard HIV testing services at a clinic while taking PrEP. In both Zambia and Uganda, the percentage of participants that reported a preference for HIV self-testing versus standard HIV testing services while on PrEP was greater in both the HIV self-testing intervention arms compared to the standard of care arm, and differences in this outcomes across randomization arms were statistically significant both in settings $(P=0.002$ Zambia; $P<0.001$ 
Table 1 Baseline demographic characteristics of the study sample

\begin{tabular}{|c|c|c|c|}
\hline & Direct HIVST distribution & HIVST coupon distribution & Standard testing \\
\hline \multicolumn{4}{|l|}{ Zambia } \\
\hline Sample Size & $N=222$ & $N=217$ & $N=194$ \\
\hline Age (med, IQR) & 24 (20 to 28$)$ & 24 (21 to 29$)$ & 24 (21 to 29$)$ \\
\hline Have a primary partner & $127(57 \%)$ & $133(61 \%)$ & $121(62 \%)$ \\
\hline Can read and write & 175 (79\%) & $176(81 \%)$ & 137 (71\%) \\
\hline Mobile phone ownership & $185(83 \%)$ & $190(88 \%)$ & $170(88 \%)$ \\
\hline \multicolumn{4}{|l|}{ Monthly income } \\
\hline No income & $48(22 \%)$ & $50(23 \%)$ & $45(\%)$ \\
\hline$<250$ kwacha $^{\mathrm{a}}$ & $21(10 \%)$ & 40 (19\%) & $28(\%)$ \\
\hline $251-500$ kwacha $^{a}$ & $58(26 \%)$ & $41(19 \%)$ & $42(\%)$ \\
\hline 501-1000 kwacha ${ }^{a}$ & $55(25 \%)$ & $57(27 \%)$ & $44(\%)$ \\
\hline $1001-1500$ kwacha $^{a}$ & $21(10 \%)$ & $15(7 \%)$ & $13(\%)$ \\
\hline$>1500$ kwacha $^{a}$ & $17(8 \%)$ & $11(5 \%)$ & $19(\%)$ \\
\hline Years in sex work (med, IQR) & $4(2$ to 8$)$ & 5 (3 to 8 ) & $3(5$ to 9$)$ \\
\hline Inconsistent condom use with clients & $169(77 \%)$ & $152(71 \%)$ & $144(76 \%)$ \\
\hline Risk of acquiring HIV in next year, 10-point scale ${ }^{\mathrm{b}}$ (med, IQR) & $6(5$ to 8$)$ & $6(5$ to 7$)$ & 5 (4 to 7$)$ \\
\hline \multicolumn{4}{|l|}{ Uganda } \\
\hline Sample size & $N=247$ & $N=240$ & $N=262$ \\
\hline Age (med, IQR) & 27.5 (24 to 31$)$ & 28 (24 to 31$)$ & 28 (24 to 32$)$ \\
\hline Have a primary partner & $160(67 \%)$ & $141(57 \%)$ & $156(60 \%)$ \\
\hline Can read and write & $209(87.1)$ & $209(84.6)$ & $228(88.0)$ \\
\hline Mobile phone ownership & $235(98 \%)$ & $233(94 \%)$ & $249(95 \%)$ \\
\hline \multicolumn{4}{|l|}{ Monthly income } \\
\hline No income & $2(1 \%)$ & $0(0 \%)$ & $0(0 \%)$ \\
\hline$<250 \cup G X^{c}$ & $50(21 \%)$ & $56(23 \%)$ & $41(16 \%)$ \\
\hline $251-500 \cup G X^{c}$ & $69(29 \%)$ & $80(33 \%)$ & $106(41 \%)$ \\
\hline $501-1000$ UGX & $88(37 \%)$ & $83(34 \%)$ & $90(35 \%)$ \\
\hline $1001-1500$ UGX & $27(11 \%)$ & $19(8 \%)$ & $22(8 \%)$ \\
\hline$>1500 \cup G X^{c}$ & $4(2 \%)$ & $5(2 \%)$ & $2(1 \%)$ \\
\hline Years in sex work (med, IQR) & 5 (3 to 8$)$ & 4 (3 to 8 ) & $5(2$ to 8$)$ \\
\hline Inconsistent condom use with clients & $104(44 \%)$ & $101(41 \%)$ & $104(40 \%)$ \\
\hline Risk of acquiring HIV in next year, 10-point scale ${ }^{\mathrm{b}}$ (med, IQR) & $4(2$ to 6$)$ & $5(3$ to 6$)$ & $4(2$ to 5$)$ \\
\hline
\end{tabular}

${ }^{a}$ Exchange rate: 1 USD = 9.2 Zambian Kwacha (September 2016)

${ }^{\mathrm{b}}$ Participants were asked to indicated how likely it is that they will contract HIV in the next year using a 10-rung ladder scale. Larger scores indicated a greater likelihood of getting HIV

'Exchange rate: 1 USD = 3366 Uganda shilling (UGX) (October 2016)

Uganda). The majority of all participants both Zambia (87\%) and Uganda (81\%), however, preferred HIV self-testing versus standard HIV testing services while on PrEP.

\section{Discussion}

FSW in both Zambian transit towns and urban Uganda reported high interest in PrEP and preferred HIV self-testing over standard HIV testing services at clinics to test for HIV regularly (every 3 months) while on
PrEP. HIV self-testing for regular, repeat testing was acceptable among FSWs in this study, especially among those who previously had the opportunity to self-test. FSW who had the opportunity to self-test previously may have preferred this approach over standard HIV testing services because they were less intimidated by the new testing technology and had a favorable experience HIV self-testing. These results indicate that HIV self-testing could be used to support PrEP delivery by moving some of the burden of HIV testing outside of 


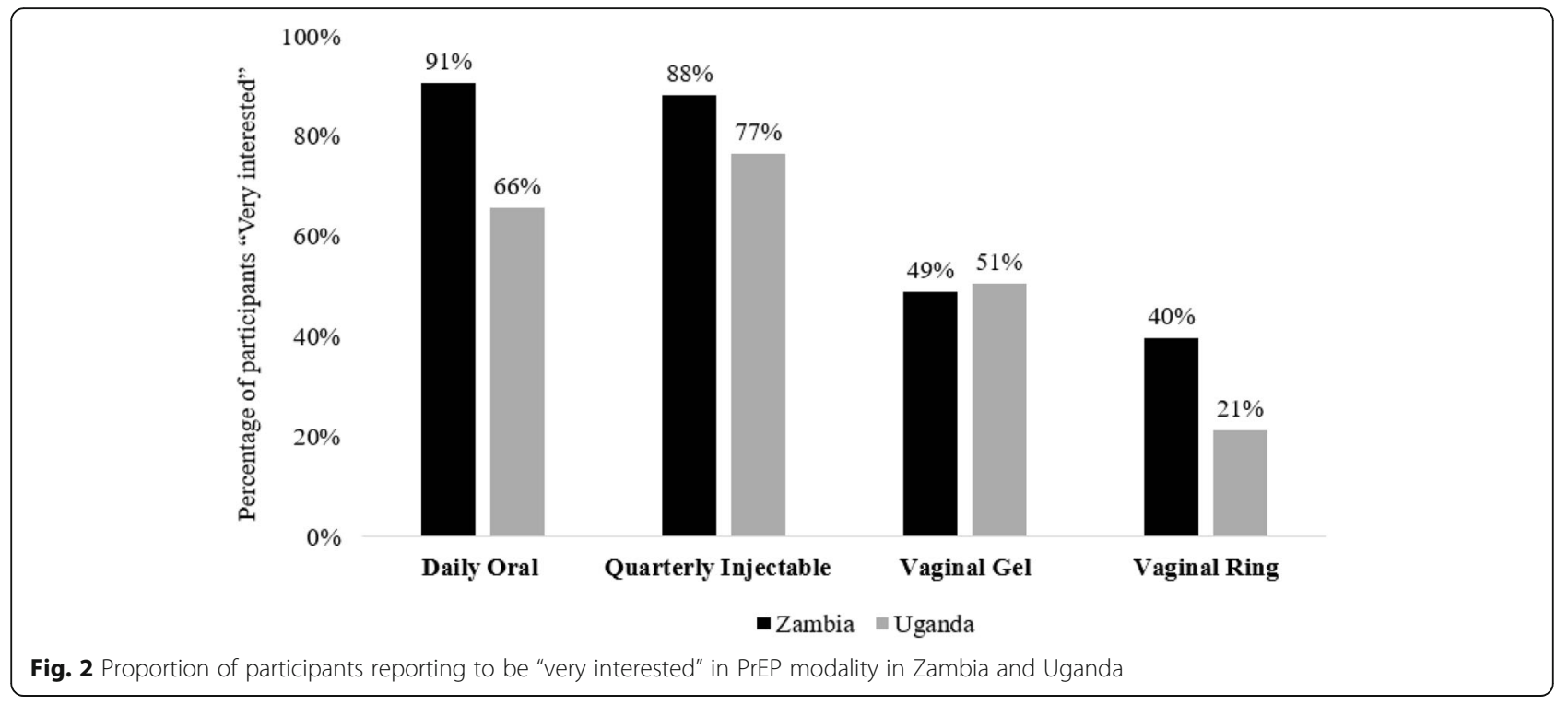

health clinics, preventing stigma and discrimination of FSW from healthcare providers and maintaining the confidentiality of FSW taking PrEP [22].

Daily oral PrEP has been shown to be acceptable [29] and implementation to be feasible [20] among other populations of FSWs. In the current study, acceptability of PrEP for prevention of HIV acquisition was high among FSWs in both Zambia and Uganda, but some PrEP modalities were more popular than others. In both study settings, FSWs preferred daily oral PrEP or quarterly injectable PrEP over PrEP in the form of a vaginal gel or ring. This preference is likely explained by the prevalence of various types of non-barrier contraceptive methods available to women in these countries. A recent study conducted among the same population of FSW in Zambia found that the majority of FSW who used a method of non-barrier contraception used injectable contraception; the second most common form of non-barrier contraception was the oral birth control pill [30]. Less than $1 \%$ of Zambian FSW in that study reported use of the vaginal ring [30]. Lack of familiarity with contraceptives inserted in the vagina might have made FSW less interested in PrEP modalities that utilized this strategy.

Acceptability of HIV self-testing was extremely high across randomization arms in both Zambia and Uganda. More than two thirds of participants in the standard of care arm preferred HIV self-testing to standard HIV testing services while taking PrEP in both settings, despite never having had the opportunity to self-test themselves. Willingness to test for HIV every three months was also near universal in both settings, thus suggesting that barriers to HIV self-testing among members of this population are likely attributable to logistics or stigma and discrimination from healthcare providers rather than a lack of will. As demonstrated in the two parent trials, HIV self-testing has the potential to help FSW overcome some of these barriers and achieve both high HIV testing coverage and high levels of repeat testing $[22,26]$. A recent study, however, suggests that FSW might have difficulty interpreting HIV self-test results [31], indicating that appropriate pre-test training and on demand support should be considered with the implementation of HIV self-testing.

This study has both strengths and limitations. We found consistent results in two diverse populations of FSWs, improving the generalizability of our findings. Participants were engaged in a randomized trial of HIV self-testing, and thus we were able to compare preferences for HIV self-testing between women who had been randomized to receive a self-test compared to those who did not receive a self-test. Questions related to acceptability of HIV self-testing were therefore not hypothetical for women who had previously had exposure to an HIV self-test. None of the participants in this study, however, actually used or had access to PrEP, and thus they might not have fully understood the research assistants' explanation of this HIV prevention intervention. Also, as a hypothetical scenario,

Table 2 Willingness to be tested for HIV every three months while taking PrEP by randomization arm

\begin{tabular}{lllll}
\hline & Direct HIVST distribution & HIVST coupon distribution & Standard testing & $P$-value \\
\hline Zambia & $217(98.2 \%)$ & $214(98.6 \%)$ & $191(99.0 \%)$ & 0.64 \\
Uganda & $232(97.1 \%)$ & $235(94.4 \%)$ & $257(96.5 \%)$ & 0.19 \\
\hline
\end{tabular}

${ }^{1}$ Estimated using mixed effects logistic regression models with a fixed effect for study arm and a random effect for peer educator group 


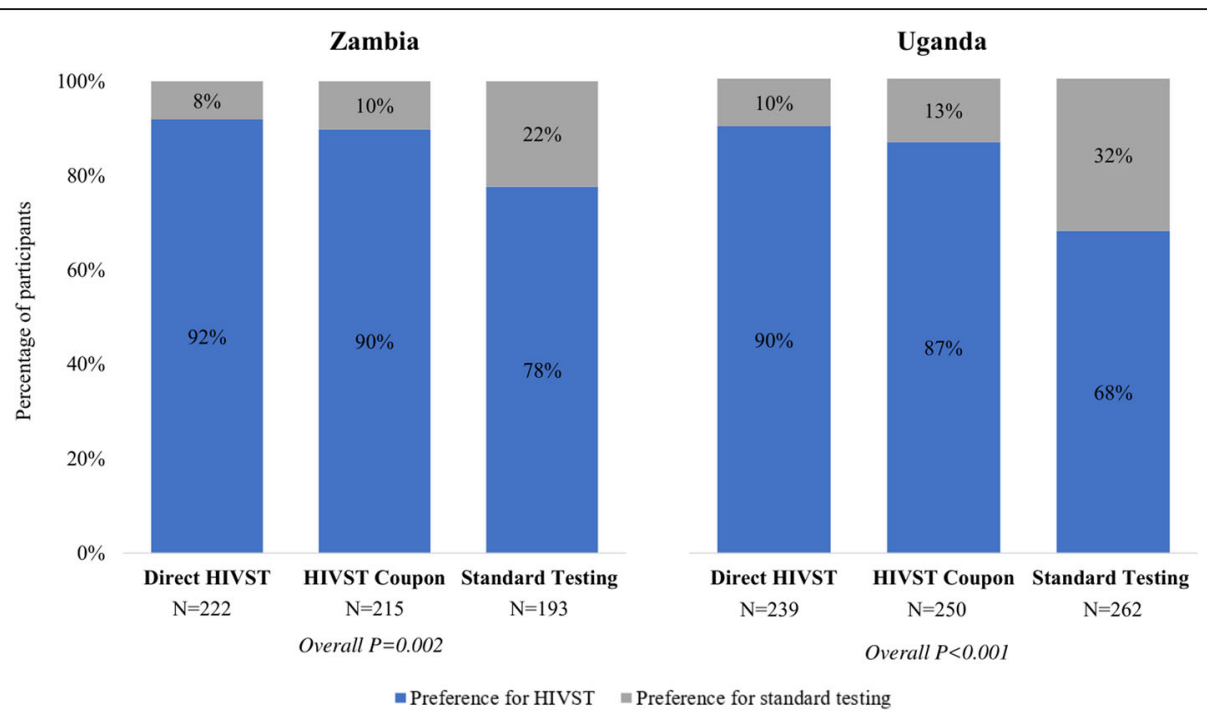

Fig. 3 Percentage of participants with a preference for HIV self-testing (HIVST) or standard HIV testing at a clinic while taking PrEP by study randomization in Zambia and Uganda

participants may be more or less likely to indicate that they would use an intervention than they would if they were actually making a decision in real life. Actual behaviors may therefore differ from anticipated behavior. Finally, our selection of study participants may have induced bias in the effect estimates because we selected on a variable (i.e., HIV-negative status) that was reported after randomization. However, PrEP is only indicated for individuals who test negative for HIV, and participants who are living with HIV may have substantially different responses to hypothetical questions regarding HIV prevention. Furthermore, to induce bias, the study randomization arm would have to causally affect HIV status. It is unlikely for this to occur in the one-month timeframe of the study.

\section{Conclusions}

In many sub-Saharan African countries, FSW are a priority population for HIV prevention interventions because of their increased risk of both HIV acquisition and transmission [16, 32]. As more sub-Saharan African countries, including Kenya, Uganda, and South Africa, begin to scale PrEP nationally, understanding preferences for PrEP and interventions for ongoing engagement in care is of increased importance. Here, we found that PrEP is highly acceptable among members of this population and that HIV self-testing is preferred over standard HIV testing services to support regular HIV testing while on PrEP. Previous studies have found HIV self-testing to be effective at increasing frequent testing among FSWs $[25,26]$. Policy makers should consider both scaling PrEP to FSW living in high prevalence areas and using HIV self-tests to support PrEP delivery and facilitate detection of breakthrough HIV infections.

\section{Acknowledgements}

We would like to acknowledge all the study participants who took time participating in this study as well as the research assistants who worked with us collecting this data.

\section{Funding}

This study was funded by the International Initiative for Impact Evaluation (3ie). KFO was additionally supported by the National Institute of Allergy and Infectious Disease (T32-Al007535, PI: Seage) and the National Institute for Mental Health (R01MH1 10296, PI: Heffron and R01-MH113572, PIs: Baeten \& Ngure). TB was funded by the Alexander von Humboldt Foundation through the Alexander von Humboldt Professorship endowed by the German Federal Ministry of Education and Research. He was also supported by the Wellcome Trust, the European Commission, the Clinton Health Access Initiative and the National Institute of Child Health and Human Development (R01-HD084233), the National Institute of Allergy and Infectious Disease (R01-Al124389 and R01-Al112339) and the Fogarty International Center (D43-TW009775). CEO was supported in part by the National Institute on Drug Abuse (T32-DA013911, PI: Flanigan) and the National Institute of Mental Health (R25-MH083620, PI: Nunn). The funders had no role in study design, data collection and analysis, decision to publish, or preparation of the manuscript.

Availability of data and materials

De-identified datasets from both studies are available through the International Initiative for Impact Evaluation's data repository (https://dataverse.harvard.edu/ dataset.xhtml?persistentld=doi:10.7910/DVN/OCWCF5 and https:// dataverse.harvard.edu/dataset.xhtml?persistentld=doi:10.7910/DVN/OVEAC9).

\section{Disclaimers}

The authors declare no conflicts of interest.

\section{Authors' contributions}

KFO and CEO conceptualized the paper, conducted the analysis, and wrote the first draft. KFO, CEO, MC, DKM, TN, MM, AN, SC, NK, CK, and TB oversaw study administration and the collection of data. All authors edited the draft and provided insights into the manuscript. All authors read and approved the final manuscript.

\section{Ethics approval and consent to participate}

Both studies were reviewed and approved by the Institutional Review Board at the Harvard T.H. Chan School of Public Health. The Zambia study was reviewed and approved by the Institutional Review Board at ERES Converge (Lusaka, Zambia) and the Uganda study was reviewed and approved by the Institutional Review Board at Mildmay (Kampala, Uganda). Written informed consent was obtained from all participants. 


\section{Consent for publication}

Not applicable.

\section{Competing interests}

The authors declare that they have no competing interests.

\section{Publisher's Note}

Springer Nature remains neutral with regard to jurisdictional claims in published maps and institutional affiliations.

\section{Author details}

'International Clinical Research Center, University of Washington, Seattle, USA. ${ }^{2} J o h n$ Snow, Inc, Lusaka, Zambia. International Research Consortium, Kampala, Uganda. ${ }^{4}$ Uganda Health Marketing Group, Kampala, Uganda. ${ }^{5}$ Department of Global Health and Population, Harvard T.H. Chan School of Public Health, Boston, USA. ${ }^{6}$ Africa Health Research Institute, KwaZulu-Natal, South Africa. ${ }^{7}$ Heidelberg Institute of Public Health, University of Heidelberg, Heidelberg, Germany. ${ }^{8}$ Francis I. Proctor Foundation, University of California San Francisco, 513 Parnassus Ave, Room S334J, San Francisco, CA, USA. ${ }^{9}$ Department of Ophthalmology, University of California, San Francisco, San Francisco, USA. ${ }^{10}$ Department of Epidemiology \& Biostatistics, University of California, San Francisco, USA.

Received: 3 July 2018 Accepted: 26 September 2018 Published online: 04 October 2018

\section{References}

1. Grant RM, Lama JR, Anderson PL, McMahan V, Liu AY, Vargas L, et al. Preexposure chemoprophylaxis for HIV prevention in men who have sex with men. N Engl J Med. 2010;363:2587-99.

2. Baeten JM, Donnell D, Ndase P, Mugo NR, Campbell JD, Wangisi J, et al. Antiretroviral prophylaxis for HIV prevention in heterosexual men and women. N Engl J Med. 2012;367:399-410.

3. Thigpen MC, Kebaabetswe PM, Paxton LA, Smith DK, Rose CE, Segolodi TM, et al. Antiretroviral Preexposure prophylaxis for heterosexual HIV transmission in Botswana. N Engl J Med. 2012;367:423-34.

4. Montgomery MC, Oldenburg CE, Nunn AS, Mena L, Anderson P, Liegler T, et al. Adherence to pre-exposure prophylaxis for HIV prevention in a clinical setting. PLoS One. 2016;11:e0157742-10.

5. Volk J, Marcus JL, Phengrasamy T, Blechinger D, Nguyen DP, Follansbee $S$, et al. No new HIV infections with increasing use of HIV preexposure prophylaxis in a clinical practice setting. Clinical infectious diseases. 2015;61:1-10.

6. Thaden JT, Gandhi M, Okochi H, Hurt CB, McKellar MS. Seroconversion on preexposure prophylaxis. AIDS. 2018;32:F1-4

7. Hurt CB, Eron JJ, Cohen MS. Pre-exposure prophylaxis and antiretroviral resistance: HIV prevention at a cost? Clin Infect Dis. 2011;53:1265-70.

8. WHO. Consolidated guidelines on the use of antiretroviral drugs for treating and preventing HIV infection. Geneva: World Health Organization; 2016.

9. Centers for Disease Control and Prevention (CDC). Preexposure prophylaxis for the prevention of HIV infection in the United States, 2014: A clinical practice guideline 2014.

10. Mack N, Odhiambo J, Wong CM, Agot K. Barriers and facilitators to preexposure prophylaxis (PrEP) eligibility screening and ongoing HIV testing among target populations in Bondo and Rarieda, Kenya: results of a consultation with community stakeholders. BMC Health Serv Res. 2014;14:231.

11. Ngure K, Heffron R, Mugo N, Thomson KA, Irungu E, Njuguna N, et al. Feasibility and acceptability of HIV self-testing among pre-exposure prophylaxis users in Kenya. J Int AIDS Soc. 2017;20:21234

12. Figueroa C, Johnson C, Ford N, Sands A, Dalal S, Meurant R, et al. Reliability of HIV rapid diagnostic tests for self-testing compared with testing by health-care workers: a systematic review and meta-analysis. The Lancet HIV. 2018;5:e277-90.

13. Figueroa C, Johnson C, Verster A, Baggaley R. Attitudes and acceptability on HIV self-testing among key populations: a literature review. AIDS Behav. 2015;19:1-17.

14. Krause J, Subklew-Sehume F, Kenyon C, Colebunders R. Acceptability of HIV self-testing: a systematic literature review. BMC Public Health. 2013;13:1-1.

15. Stevens DR, Vrana CJ, Dlin RE, Korte JE. A global review of HIV self-testing: themes and implications. AIDS Behav. 2017;22:1-16.
16. Baral S, Beyrer C, Muessig K, Poteat T, Wirtz AL, Decker MR, et al. Burden of HIV among female sex workers in low-income and middle-income countries: a systematic review and meta-analysis. Lancet Infect Dis. 2012;12:538-49.

17. Beyrer C, Crago A-L, Bekker L-G, Butler J, Shannon K, Kerrigan D, et al. An action agenda for HIV and sex workers. Lancet. 2015;385:287-301.

18. Deering KN, Lyons T, Feng CX, Nosyk B, Strathdee SA, Montaner JSG, et al. Client demands for unsafe sex: the socioeconomic risk environment for HIV among street and off-street sex workers. JAIDS Journal of Acquired Immune Deficiency Syndromes. 2013;63:522-31.

19. Cowan FM, Delany-Moretlwe S. Promise and pitfalls of pre-exposure prophylaxis for female sex workers. Curr Opin HIV AIDS. 2016;11:27-34.

20. Eakle R, Gomez GB, Naicker N, Bothma R, Mbogua J, Cabrera Escobar MA, et al. HIV pre-exposure prophylaxis and early antiretroviral treatment among female sex workers in South Africa: results from a prospective observational demonstration project. PLoS Med. 2017:14:e1002444-17.

21. Dudina VI, Bowling JM, King EJ, Dudina VI, Moracco KE. Motivators and barriers to HIV testing among street-based female sex workers in St. Petersburg, Russia. Global Public Health. 2015;0:1-16.

22. Chanda MM, Perez-Brumer AG, Ortblad KF, Mwale M, Chongo S, Kamungoma $\mathrm{N}$, et al. Barriers and facilitators to HIV testing among Zambian female sex Workers in Three Transit Hubs. AIDS Patient Care STDs. 2017;31:290-6.

23. Lancaster KE, Cernigliaro D, Zulliger R, Fleming PF. HIV care and treatment experiences among female sex workers living with HIV in sub-Saharan Africa: a systematic review. Afr J AIDS Res. 2016;15:377-86.

24. Wanyenze RK, Musinguzi G, Kiguli J, Nuwaha F, Mujisha G, Musinguzi J, et al "When they know that you are a sex worker, you will be the last person to be treated": perceptions and experiences of female sex workers in accessing HIV services in Uganda. BMC Int Health Hum Rights. 2017;17:11.

25. Chanda MM, Ortblad KF, Mwale M, Chongo S, Kanchele C, Kamungoma N, et al. HIV self-testing among female sex workers in Zambia: a cluster randomized controlled trial. PLoS Med. 2017:e1002442:14

26. Ortblad K, Kibuuka Musoke D, Ngabirano T, Nakitende A, Magoola J, Kayiira $P$, et al. Direct provision versus facility collection of HIV self-tests among female sex workers in Uganda: a cluster-randomized controlled health systems trial. PLoS Med. 2017;14:e1002458-24

27. Oldenburg CE, Ortblad KF, Chanda M, Mwanda K, Nicodemus W, Sikaundi R, et al. Zambian peer educators for HIV self-testing (ZEST) study: rationale and design of a cluster randomized trial of HIV self-testing among female sex workers in Zambia. BMJ Open. 2017:20:e014780.

28. Zachary D, Mwenge L, Muyoyeta M, Shanaube K, Schaap A, Bond V, et al. Field comparison of OraQuick ADVANCE rapid HIV-1/2 antibody test and two bloodbased rapid HIV antibody tests in Zambia. BMC Infect Dis. 2012;12:183.

29. Eakle R, Bourne A, Mbogua J, Mutanha N, Rees H. Exploring acceptability of oral PrEP prior to implementation among female sex workers in South Africa. J Int AIDS Soc. 2018;21:e25081.

30. Chanda MM, Ortblad KF, Mwale M, Chongo S, Kanchele C, Kamungoma N et al. Contraceptive use and unplanned pregnancy among female sex workers in Zambia. Contraception. 2017:96:1-23.

31. Ortblad KF, Musoke DK, Ngabirano T, Nakitende A, Haberer JE, McConnell M, et al. Female sex workers often incorrectly interpret HIV self-test results in Uganda. J Acquir Immune Defic Syndr. 2018;79:e42-5.

32. Shannon K, Strathdee SA, Goldenberg SM, Duff P, Mwangi P, Rusakova M, et al. Global epidemiology of HIV among female sex workers: influence of structural determinants. Lancet. 2015;385:55-71.

Ready to submit your research? Choose BMC and benefit from:

- fast, convenient online submission

- thorough peer review by experienced researchers in your field

- rapid publication on acceptance

- support for research data, including large and complex data types

- gold Open Access which fosters wider collaboration and increased citations

- maximum visibility for your research: over $100 \mathrm{M}$ website views per year

At BMC, research is always in progress.

Learn more biomedcentral.com/submission 